\title{
Regioselectivity in the multicomponent reaction of 5-aminopyrazoles, cyclic 1,3-diketones and dimethyl- formamide dimethylacetal under controlled microwave heating
}

\author{
Kamal Usef Sadek ${ }^{* 1}$, Ramadan Ahmed Mekheimer ${ }^{1,2}$, \\ Tahany Mahmoud Mohamed ${ }^{1}$, Moustafa Sherief Moustafa*3 \\ and Mohamed Hilmy Elnagdi ${ }^{3}$
}

\section{Full Research Paper}

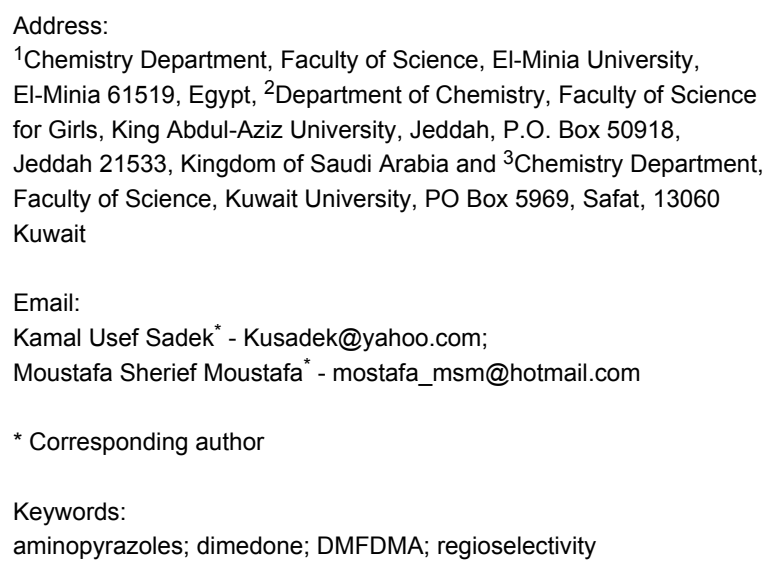

${ }^{1}$ Chemistry Department, Faculty of Science, El-Minia University, El-Minia 61519, Egypt, ${ }^{2}$ Department of Chemistry, Faculty of Science for Girls, King Abdul-Aziz University, Jeddah, P.O. Box 50918, Jeddah 21533, Kingdom of Saudi Arabia and ${ }^{3}$ Chemistry Department, Faculty of Science, Kuwait University, PO Box 5969, Safat, 13060 Kuwait

Keywords:

aminopyrazoles; dimedone; DMFDMA; regioselectivity

\begin{abstract}
The multicomponent reaction of 5-aminopyrazole derivatives with cyclic 1,3-dicarbonyl compounds and dimethylformamide dimethylacetal (DMFDMA) in DMF at $150{ }^{\circ} \mathrm{C}$ under controlled microwave heating afforded regioselectively 8,9dihydropyrazolo[1,5-a]quinazolin-6(7H)-ones 6 rather than the corresponding dihydropyrazolo[5,1-b]quinazolin-8(5H)-ones 4 .
\end{abstract}

\section{Introduction}

Several naturally occurring and synthetic compounds containing quinazoline derivatives are of considerable interest in fields related to the organic and medicinal chemistry of natural products $[1,2]$. The quinazoline ring system represents the core skeleton of an important class of heterocyclic compounds possessing a wide range of biological activities [3,4]. Multicomponent reactions (MCR) occupy an interesting position in organic synthesis because of their atom economy, simple procedures and convergent character [5-7]. An unresolved issue in multicomponent reactions is whether their selectivity is chemo- 
or regioselectivity, or both, due to the several possible parallel reaction pathways, which result in the formation of different products [8-10]. Many factors modulate the selectivity of synthetic transformations, such as temperature, pressure, solvent, catalyst and type of reaction control, i.e., either kinetic or thermodynamic [11-13]. It has been reported that the use of microwave or ultrasound irradiation provides an additional parameter for synthetic selectivity [14-17].

\section{Results and Discussion}

The multicomponent reaction of 5-aminopyrazoles, dimedone and aromatic aldehydes was reported to afford several different tricyclic products. Thus, in an early report [18], the reaction of the three components in ethanol under conventional heating afforded mainly the corresponding pyrazolo[3,4-b]quinolin-5ones. This finding was later supported by other authors [19]. Recently, the results of an interesting study dealing with such reactions were described by Chebanov et al. [20] Specifically, these researchers performed the reaction at $150{ }^{\circ} \mathrm{C}$ in the presence of triethylamine by employing a sealed vessel under microwave or conventional heating, and which thus afforded pyrazoloquinolinones (Hantzsch-type dihydropyridines). On the other hand, the use of sonication at room temperature under neutral conditions favours the formation of isomeric pyrazolo[5,1-b]quinazolin-8(4H)-ones (Biginelli-type dihydropyrimidines) [9]. Employing more nucleophilic bases to catalyse the reaction afforded the corresponding pyrazolo[4,3c] quinazolin-9-ones [20]. It was concluded that, under ambient and neutral conditions, the reaction proceeds under kinetic control, and the Biginelli-type dihydropyrimidines are the predominant isomers. Increasing the reaction temperature in the presence of triethylamine as base produces the more thermodynamically stable dihydropyridine (Hantzsch-type product). In addition, the nature of the catalyst plays an important role [20].
A one-pot three component reaction of 5-amino- $1 H$-pyrazole-4carbonitrile, dimedone and triethylorthoesters in toluene under reflux was recently reported to afford the corresponding pyrazolo[1,5-a]-quinazolin-6-one derivatives [21]. Although it is well established that 5-amino-pyrazoles have nonequivalent nucleophilic reaction centres in the aminopyrazole scaffold (N1, $\mathrm{C} 4, \mathrm{NH}_{2}$ ), which can lead to the formation of several different tricyclic reaction products, no general basis on which to determine the preferred tautomeric form of the final product has been established.

In continuation of our studies in which we performed multicomponent reactions using controlled microwave heating [22-24], we report herein the results of our investigation concerning the regioselectivity in multicomponent reactions of 5-aminopyrazoles, cyclic 1,3-diketones and dimethylformamide dimethylacetal (DMFDMA) under controlled microwave heating.

We began this study by treating 5-amino-3-methylpyrazole (1a) and dimedone (2a) with DMFDMA (3) in DMF under microwave heating at $150{ }^{\circ} \mathrm{C}$ for $15 \mathrm{~min}$. After being cooled to room temperature, the precipitated solid product was isolated in $88 \%$ yield (Table 1 ). The mass spectrum of the reaction product showed a molecular ion peak $m / z=229.12(100 \%)$. The ${ }^{1} \mathrm{H}$ NMR revealed a singlet signal at $\delta=6.70 \mathrm{ppm}$ integrated for one proton, which was assigned to the pyrazoloquinazolone $\mathrm{C}_{3}$ proton, and which indicates the lack of involvement of such a proton in the condensation leading to the tricyclic system. Although, it was previously reported [20] that, due to reduced steric hindrance, the multicomponent reaction of 5-amino-3methyl-pyrazole, aromatic aldehydes and dimedone under controlled microwave irradiation at $150{ }^{\circ} \mathrm{C}$ involves the participation of $\mathrm{C}_{3}-\mathrm{H}$ of the pyrazole ring in such a cyclocondensation reaction, this is not favoured in our case. In addition two

\begin{tabular}{|c|c|c|c|c|c|}
\hline entry & compound & 5-aminopyrazole, $\mathbf{1}$ & cyclic 1,3-diketone, $\mathbf{2}$ & product & yield $(\%)$ \\
\hline 1 & $1 a$ & $\begin{array}{l}\mathrm{R}=\mathrm{CH}_{3}, \\
\mathrm{R}^{1}=\mathrm{H}\end{array}$ & $\mathbf{2 a} ; \mathrm{R}^{2}=\mathrm{CH}_{3}$ & $6 a$ & 88 \\
\hline 2 & $1 a$ & $\begin{array}{l}\mathrm{R}=\mathrm{CH}_{3}, \\
\mathrm{R}^{1}=\mathrm{H}\end{array}$ & $\mathbf{2 b} ; \mathrm{R}^{2}=\mathrm{H}$ & $6 b$ & 85 \\
\hline 3 & $1 \mathrm{~b}$ & $\begin{array}{l}\mathrm{R}=\mathrm{NH}_{2}, \\
\mathrm{R}^{1}=\mathrm{CO}_{2} \mathrm{Et}\end{array}$ & $2 b ; R^{2}=H$ & $6 c$ & 89 \\
\hline 4 & 1c & $\begin{array}{l}\mathrm{R}=\mathrm{CH}_{3}, \\
\mathrm{R}^{1}=\mathrm{C}_{6} \mathrm{H}_{5}\end{array}$ & 2a; $\mathrm{R}^{2}=\mathrm{CH}_{3}$ & $6 d$ & 83 \\
\hline 5 & $1 d$ & $\begin{array}{l}\mathrm{R}=\mathrm{C}_{6} \mathrm{H}_{5} \\
\mathrm{R}^{1}=\mathrm{H}\end{array}$ & $\mathbf{2 b} ; \mathrm{R}^{2}=\mathrm{H}$ & $6 e$ & 82 \\
\hline 6 & $1 e$ & $\begin{array}{l}\mathrm{R}=\mathrm{C}_{6} \mathrm{H}_{5} \\
\mathrm{R}^{1}=\mathrm{H}\end{array}$ & $2 \mathrm{a} ; \mathrm{R}^{2}=\mathrm{CH}_{3}$ & $6 f$ & 83 \\
\hline 7 & $1 f$ & $\begin{array}{l}\mathrm{R}=\mathrm{OH} \\
\mathrm{R}^{1}=\mathrm{C}_{6} \mathrm{H}_{5} \mathrm{~N}=\mathrm{N}-\end{array}$ & $\mathbf{2 a} ; \mathrm{R}^{2}=\mathrm{CH}_{3}$ & $6 \mathrm{~g}$ & 84 \\
\hline
\end{tabular}




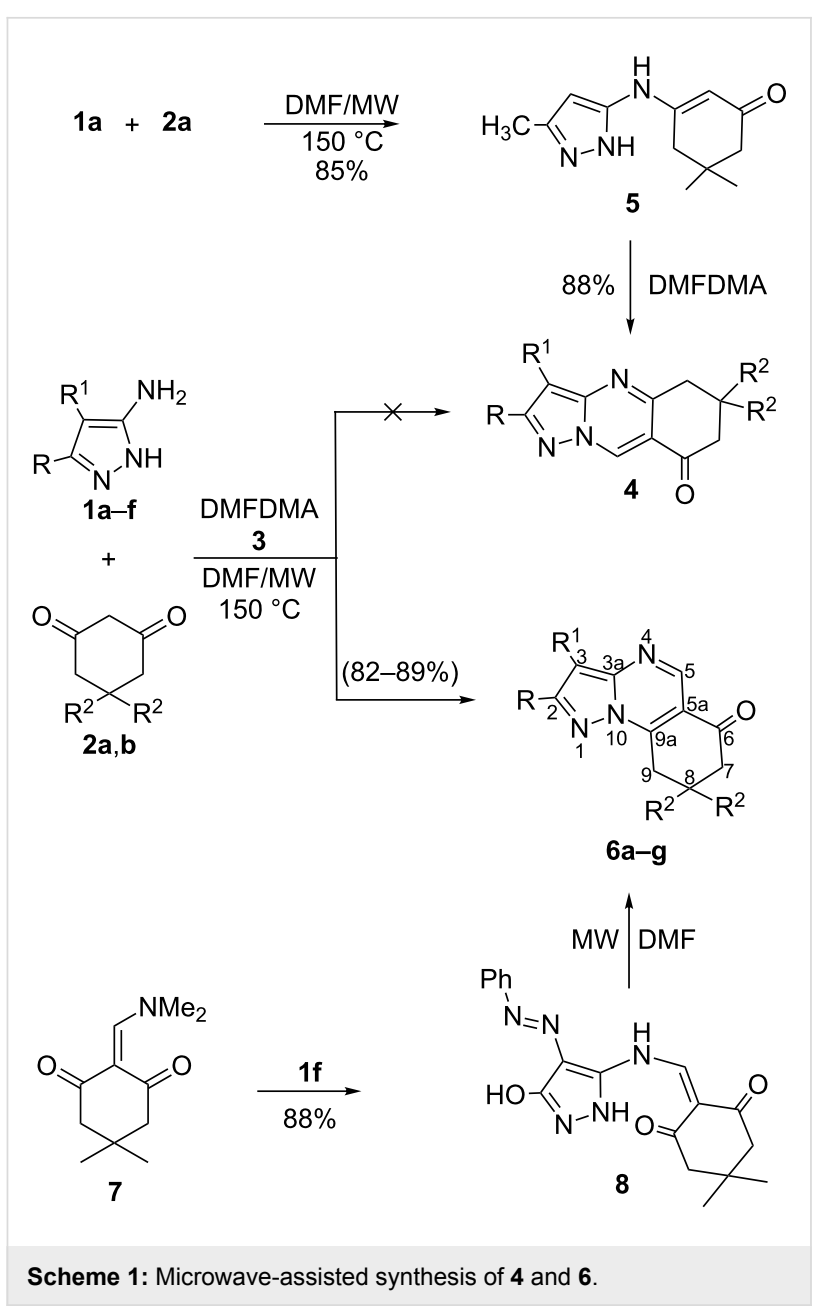

signals were assigned to two $\mathrm{CH}_{2}$ groups and three methyl functions, and a singlet at $\delta=8.75 \mathrm{ppm}$ corresponding to one proton at $\mathrm{C}_{5}$. The pyrazolo[1,5-a]-quinazolin- $8(5 H)$-one $\mathbf{6 a}$ was established as the reaction product, and ${ }^{13} \mathrm{C} N M R$ was in agreement with the proposed structure, rather than with isomeric $\mathbf{4 a}$, which was prepared by first reacting 1a with dimedone (2a) in DMF under microwave heating at $150{ }^{\circ} \mathrm{C}$ for $10 \mathrm{~min}$ to afford $\mathbf{5}$. Subsequently, treating compound $\mathbf{5}$ with DMFDMA (3), under the same experimental conditions, gave compound $\mathbf{6 a}$ in excellent yield (Scheme 1 and Table 1). Furthermore, the structures of compounds $\mathbf{5}$ and $\mathbf{6 a}$ were unambiguously confirmed by single-crystal X-ray diffraction [25,26] (Figure 1, Figure 2 and Table 1, Table 2, Table 3).

With this result in hand, we went on to study the scope of such multicomponent reactions with several substituted 5-aminopyrazoles and cyclic 1,3-diketones. Thus, the reaction of $\mathbf{1 b}-\mathbf{f}$ with 2a,b and $\mathbf{3}$, under the same experimental conditions, afforded the corresponding pyrazolo[5,1-b]quinazolin-8(5H)-ones $6 \mathbf{b}-\mathbf{g}$, respectively. The structures of $\mathbf{6} \mathbf{b}-\mathbf{g}$ were deduced from their ${ }^{1} \mathrm{H}$ NMR, ${ }^{13} \mathrm{C}$ NMR, mass spectra and elemental analyses.

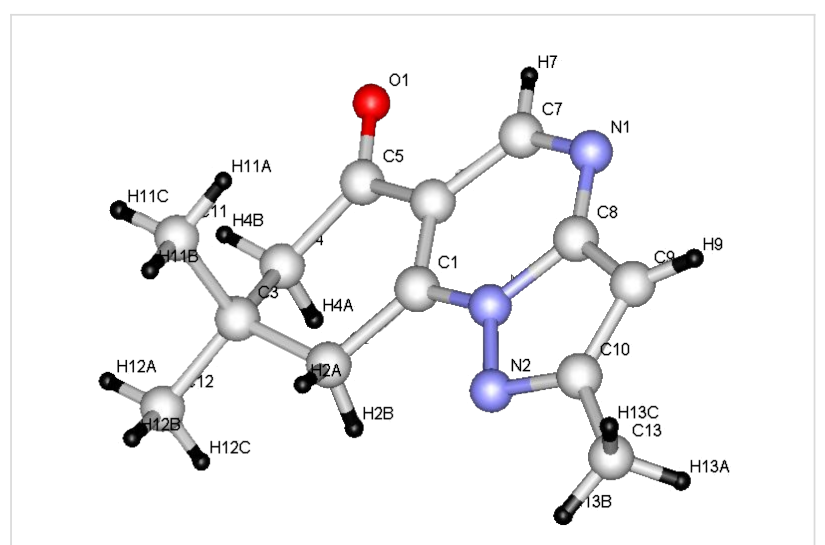

Figure 1: ORTEP diagram of compound $\mathbf{6 a}$

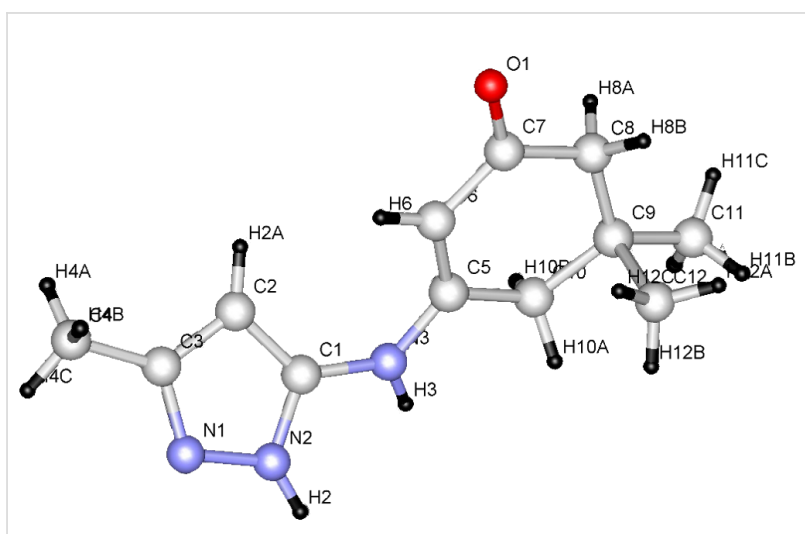

Figure 2: ORTEP diagram of compound 5.

Compound $6 \mathrm{~g}$ was also obtained by an alternative route: Compound 8 was prepared by reacting enaminone 7 with 5-aminopyrazole derivative $\mathbf{1 f}$ in DMF under microwave heating at $150{ }^{\circ} \mathrm{C}$ for $2 \mathrm{~min}$ (Table 1). When this compound was refluxed in DMF under microwave heating for $13 \mathrm{~min}$ it under-

Table 2: Selected bond lengths and bond angles for compound $\mathbf{6 a}$

\begin{tabular}{|llll}
\multicolumn{2}{c}{ bond lengths } & \multicolumn{2}{c}{ bond angles } \\
\hline $\begin{array}{l}\text { atom } \\
\text { numbers }\end{array}$ & $\begin{array}{l}\text { geometric } \\
\text { parameter } \\
(\AA)\end{array}$ & $\begin{array}{l}\text { atom } \\
\text { numbers }\end{array}$ & $\begin{array}{l}\text { geometric } \\
\text { parameter }\left(^{\circ}\right)\end{array}$ \\
& & & \\
\hline N1-C8 & $1.372(3)$ & C7-N1-C8 & $116.15(19)$ \\
N1-C7 & $1.309(3)$ & N2-N3-C1 & $125.03(16)$ \\
N2-C10 & $1.344(3)$ & C1-N3-C8 & $122.51(18)$ \\
N3-C8 & $1.397(3)$ & N3-C1-C6 & $116.10(17)$ \\
N3-C1 & $1.490(3)$ & C8-C9-C10 & $106.29(17)$ \\
N1-C6 & $1.377(3)$ & C1-C6-C5 & $119.42(19)$ \\
N6-C7 & $1.421(3)$ & N1-C7-C6 & $124.5(3)$ \\
& & N1-C8-C9 & $133.29(19)$ \\
& & N3-N2-C10 & $103.65(17)$ \\
& & N2-N3-C8 & $112.41(16)$ \\
& & N1-C8-N3 & $121.56(18)$
\end{tabular}


Table 3: Selected bond lengths and bond angles for compound $\mathbf{6 e}$

\begin{tabular}{llll}
\multicolumn{2}{c}{ bond lengths } & \multicolumn{2}{c}{ bond angles } \\
$\begin{array}{l}\text { atoms } \\
\text { numbers }\end{array}$ & $\begin{array}{l}\text { geometric } \\
\text { parameter } \\
(\AA)\end{array}$ & $\begin{array}{l}\text { atom } \\
\text { numbers }\end{array}$ & $\begin{array}{l}\text { geometric } \\
\text { parameter }\left(^{\circ}\right)\end{array}$ \\
& & & \\
\hline N3-C9 & $1.360(3)$ & C8-N3-C9 & $116.10(19)$ \\
N3-C8 & $1.3147(3)$ & N1-N2-C2 & $124.94(19)$ \\
N1-C1 & $1.346(3)$ & N2-C1-C3 & $124.71(18)$ \\
N2-C9 & $1.396(3)$ & N2-C2-C7 & $116.23(18)$ \\
N2-C2 & $1.364(3)$ & C1-C10-C9 & $120.9(17)$ \\
C2-C7 & $1.363(3)$ & C2-C7-C8 & $124.7(2)$ \\
C7-C8 & $1.428(3)$ & N3-C8-C7 & $105.78(17)$ \\
& & N3-C9-C10 & $133.37(19)$ \\
& & N2-N1-C1 & $103.94(14)$ \\
& & N1-N2-C9 & $112.01(15)$ \\
& & N2-C9-N3 & $120.99(18)$
\end{tabular}

went cyclization to give $\mathbf{6 g}$ (Scheme 1). Moreover, the structure of compounds $\mathbf{6} \mathbf{b}-\mathbf{g}$ was unequivocally established by single-crystal X-ray diffraction of compounds $\mathbf{6 e , g}$ (Figure 3, Figure 4 and Table 3, Table 4) [27,28].

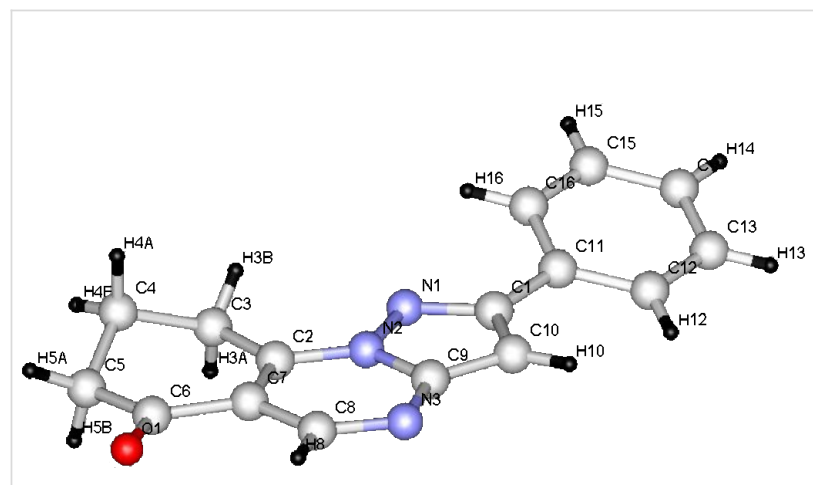

Figure 3: ORTEP diagram of compound $6 \mathbf{e}$.

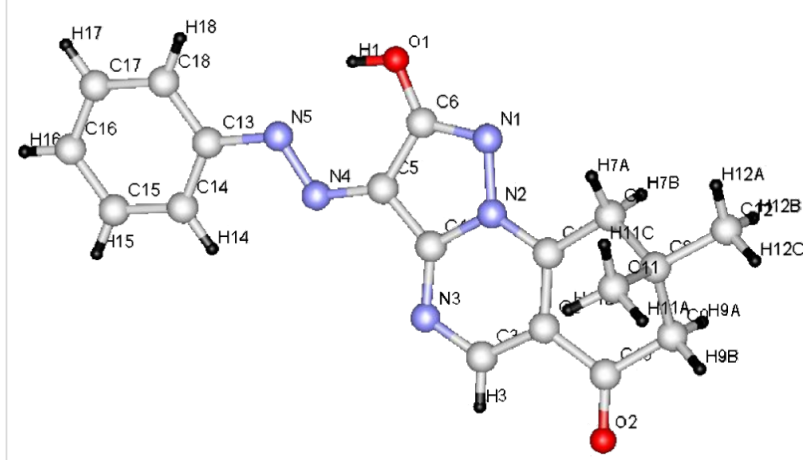

Figure 4: ORTEP diagram of compound $\mathbf{6 g}$.
Table 4: Selected bond lengths and bond angles for compound $\mathbf{6 g}$.

\begin{tabular}{llll}
\multicolumn{3}{c}{ bond lengths } & \multicolumn{2}{c}{ bond angles } \\
\hline $\begin{array}{l}\text { atoms } \\
\text { numbers }\end{array}$ & $\begin{array}{l}\text { geometric } \\
\text { parameter } \\
(\AA)\end{array}$ & $\begin{array}{l}\text { atom } \\
\text { numbers }\end{array}$ & $\begin{array}{l}\text { geometric } \\
\text { parameter }\left(^{\circ}\right)\end{array}$ \\
& & & \\
\hline N3-C4 & $1.330(2)$ & C3-N3-C4 & $116.18(10)$ \\
N3-C3 & $1.321(19)$ & N1-N2-C1 & $124.04(12)$ \\
N1-C6 & $1.343(17)$ & C1-N2-C4 & $121.41(12)$ \\
N2-C4 & $1.393(18)$ & N2-C1-C2 & $116.52(13)$ \\
N2-C1 & $1.343(19)$ & C4-C5-C6 & $105.52(13)$ \\
C1-C7 & $1.491(2)$ & C1-C2-C10 & $119.58(13)$ \\
C1-C2 & $1.394(2)$ & N3-C3-C2 & $123.90(14)$ \\
& & N3-C4-C5 & $132.56(14)$ \\
& & N2-N1-C6 & $104.27(11)$ \\
& & N1-N2-C4 & $114.50(11)$ \\
& & N2-C4-N3 & $123.02(13)$
\end{tabular}

A proposed mechanism to account for the formation of products 6 is illustrated in Scheme 2. The base-catalyzed reaction of cyclic 1,3-diketones 2 with DMFDMA 3 gave the enaminone 7, which subsequently reacted with 5-aminopyrazole $\mathbf{1}$ at the

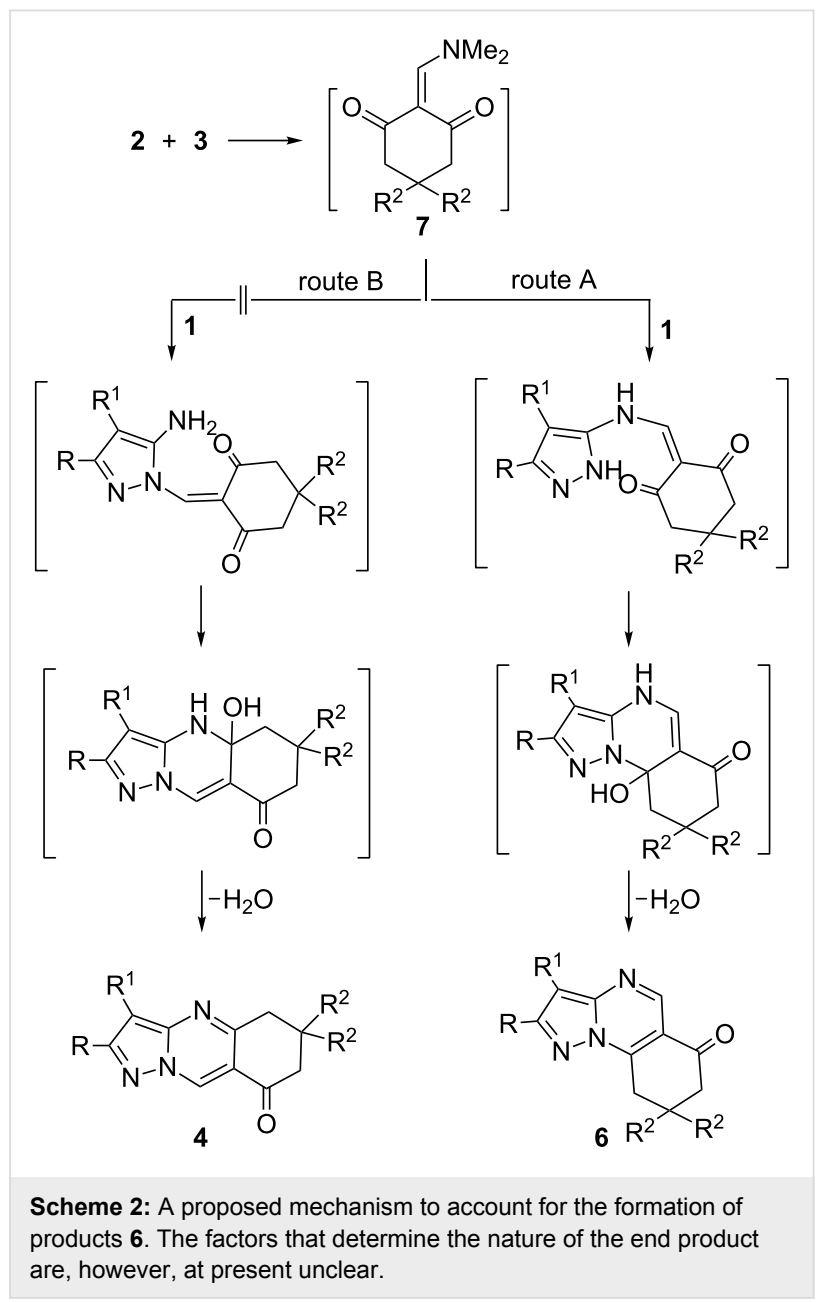


exocyclic amino function, followed by cyclization through water loss to give 6 (route A). Formation of isomeric product 4 , which would be formed by route $\mathrm{B}$, was ruled out based on spectral and X-ray diffraction data.

From the data of the X-ray crystal structure it can be concluded that the bridged head nitrogen has bond angles closer to those of $\mathrm{sp}^{3}$ nitrogen. One may thus conclude that the lone pair on this nitrogen atom does not contribute much to the actual state of the molecule and that charge-separated ions also do not contribute significantly; although, the pyrazolo[ $[5,1-b]$ quinazolin ring is almost planar.

\section{Conclusion}

In summary, we can reveal that the reaction of substituted 5-aminopyrazoles, cyclic 1,3-diketones and dimethyformamide dimethylacetal (DMFDMA, 3) proceeds by initial attack of the exocyclic amino function. Although an attack by the ring nitrogen has been proposed for the reaction of 5-aminopyrazoles with acrylonitrile [29], here steric factors hinder such an attack and the reaction occurs exclusively, in every case studied, at the amino function.

\section{Experimental}

General information. All the reactions were carried out in a Milestone START Microwave Labstation (temperature control by IR sensor). ${ }^{1} \mathrm{H}$ NMR (400 MHz) and ${ }^{13} \mathrm{C}$ NMR (100 MHz) spectra were measured on a Bruker DPX instrument by using DMSO- $d_{6}$ as solvent and TMS as internal standard. Chemical shifts are expressed as $\delta$ in ppm. Coupling constants $(J)$ are given in Hertz $(\mathrm{Hz})$. The melting points were measured in a Gallenkamp melting-point apparatus and are not corrected. Mass spectra were measured by using VG Autospec Q MS 30 and MS 9 (AEI) spectrometer with the EI $(70 \mathrm{eV})$ mode.

\section{General procedure for the synthesis of pyra- zoloquinazolinones $(6 \mathrm{a}-\mathrm{g})$}

A solution of 5-aminopyrazole derivative $\mathbf{1 a}-\mathbf{f}(1 \mathrm{mmol})$, cyclic 1,3-diketones $(\mathbf{2 a}, \mathbf{b})(1 \mathrm{mmol})$ and dimethylformamide dimethylacetal (DMFDMA, 3) (1 mmol) in DMF $(10 \mathrm{~mL})$ was heated under reflux in a Milestone Microwave Labstation at 150 ${ }^{\circ} \mathrm{C}$ for $15 \mathrm{~min}$. After concentration and cooling to room temperature, the resulting solid product so formed was collected by filtration, washed well with EtOH, dried and recrystallized from $\mathrm{EtOH}$.

2,8,8-Trimethyl-8,9-dihydropyrazolo $[5,1-b] q u i n a z o l i n-$ 6(7H)-one (6a): Greenish yellow plates, $201 \mathrm{mg}$ (88\% yield); mp $134-135{ }^{\circ} \mathrm{C} ;{ }^{1} \mathrm{H}$ NMR (400 MHz, DMSO- $\left.d_{6}\right) \delta 1.12$ (s, 6H, $\left.2 \mathrm{CH}_{3}\right), 2.48\left(\mathrm{~s}, 3 \mathrm{H}, \mathrm{CH}_{3}\right), 2.56\left(\mathrm{~s}, 2 \mathrm{H}, \mathrm{CH}_{2}\right.$ at $\left.\mathrm{C}-9\right), 3.32(\mathrm{~s}, 2 \mathrm{H}$, $\mathrm{CH}_{2}$ at $\left.\mathrm{C}-7\right), 6.70(\mathrm{~s}, 1 \mathrm{H}, \mathrm{CH}$ at $\mathrm{C}-3), 8.75(\mathrm{~s}, 1 \mathrm{H}, \mathrm{CH}$ at $\mathrm{C}-5)$;
${ }^{13} \mathrm{C}$ NMR $\left(100 \mathrm{MHz}, \mathrm{DMSO}-d_{6}\right) \delta 14.55,27.89,32.36,36.46$, 38.87, 50.08, 98.04, 112.39, 146.03, 149.34, 152.21, 157.52, 194.82; EIMS $m / z$ : $229.1\left(\mathrm{M}^{+}\right), 214,173$, calcd. for $\mathrm{C}_{13} \mathrm{H}_{15} \mathrm{~N}_{3} \mathrm{O}$ 229.28; Anal. calcd for $\mathrm{C}_{13} \mathrm{H}_{15} \mathrm{~N}_{3} \mathrm{O}: \mathrm{C}, 68.1 ; \mathrm{H}, 6.59 ; \mathrm{N}, 18.33$; found: C, $68.22 ; \mathrm{H}, 6.62 ; \mathrm{N}, 18.35 \%$.

2-Methyl-8,9-dihydropyrazolo[5,1-b]quinazolin-6(7H)-one (6b): Yellow plates, $170 \mathrm{mg}$ (85\% yield); mp $154-155{ }^{\circ} \mathrm{C} ;{ }^{1} \mathrm{H}$ NMR (400 MHz, DMSO- $\left.d_{6}\right) \delta 2.21-2.27\left(\mathrm{~m}, 2 \mathrm{H}, \mathrm{CH}_{2}\right.$ at $\left.\mathrm{C}-8\right)$, $2.66\left(\mathrm{t}, J=6.8 \mathrm{~Hz}, 2 \mathrm{H}, \mathrm{CH}_{2}\right.$ at $\left.\mathrm{C}-9\right), 3.40(\mathrm{t}, J=6.4 \mathrm{~Hz}, 2 \mathrm{H}$, $\mathrm{CH}_{2}$ at $\left.\mathrm{C}-7\right), 6.71(\mathrm{~s}, 1 \mathrm{H}, \mathrm{CH}$ at $\mathrm{C}-3), 8.77(\mathrm{~s}, 1 \mathrm{H}, \mathrm{CH}$ at $\mathrm{C}-5)$; ${ }^{13} \mathrm{C}$ NMR $\left(100 \mathrm{MHz}, \mathrm{DMSO}-d_{6}\right) \delta 14.53,19.95,33.37,36.54$, 97.91, 113.3, 146.3, 149.0, 153.9, 157.42, 194.81; EIMS m/z $201.12\left(\mathrm{M}^{+}\right)$, calcd for $\mathrm{C}_{11} \mathrm{H}_{11} \mathrm{~N}_{3} \mathrm{O} 201.22$; Anal. calcd for $\mathrm{C}_{11} \mathrm{H}_{11} \mathrm{~N}_{3} \mathrm{O}$ : C, 65.66; H, 5.51; N, 20.88; found: C, 65.68; $\mathrm{H}$, 5.49; N, 20.67\%.

Ethyl 2-amino-6-oxo-6,7,8,9-tetrahydropyrazolo [5,1b]quinazolin-3-carboxylate (6c): Yellow crystals, $243 \mathrm{mg}$ (89\% yield); mp $184-185{ }^{\circ} \mathrm{C} ;{ }^{1} \mathrm{H}$ NMR (400 MHz, DMSO- $d_{6}$ ) $\delta 1.31\left(\mathrm{t}, J=7.2 \mathrm{~Hz}, 3 \mathrm{H}, \mathrm{CH}_{3}\right), 2.10-2.20\left(\mathrm{~m}, 2 \mathrm{H}, \mathrm{CH}_{2}\right.$ at $\left.\mathrm{C}-8\right)$, $2.63\left(\mathrm{t}, J=6.8 \mathrm{~Hz}, 2 \mathrm{H}, \mathrm{CH}_{2}\right.$ at $\left.\mathrm{C}-9\right), 3.25(\mathrm{t}, J=6.8 \mathrm{~Hz}, 2 \mathrm{H}$, $\mathrm{CH}_{2}$ at C-7), 4.31 (q, $J=6.8 \mathrm{~Hz}, 2 \mathrm{H}, \mathrm{CH}_{2}$ ), 6.7 (br s, 2H, $\mathrm{NH}_{2}$ ), $8.82(\mathrm{~s}, 1 \mathrm{H}, \mathrm{CH}$ at $\mathrm{C}-5)$; EIMS $m / z 274.1\left(\mathrm{M}^{+}\right), 228,174.1$, calcd for $\mathrm{C}_{13} \mathrm{H}_{14} \mathrm{~N}_{4} \mathrm{O}_{3} 274.28$; Anal. calcd for $\mathrm{C}_{13} \mathrm{H}_{14} \mathrm{~N}_{4} \mathrm{O}_{3}$ : C, 56.93; H, 5.14; 20.43; found: C, 57.12; H, 5.23; N, 20.45\%

2,8,8-Trimethyl-3-phenyl-8,9-dihydropyrazolo[5,1-b]quinazolin-6(7H)-one (6d): Pale yellow crystals, $253 \mathrm{mg}(83 \%$ yield); mp 279-280 ${ }^{\circ} \mathrm{C} ;{ }^{1} \mathrm{H}$ NMR (400 MHz, DMSO- $d_{6}$ ) $\delta 1.15$ (s, 6H, $\left.2 \mathrm{CH}_{3}\right), 2.49$ (s, 2H, $\mathrm{CH}_{2}$ at C-9), $2.58\left(\mathrm{~s}, 3 \mathrm{H}, \mathrm{CH}_{3}\right.$ at $\mathrm{C}-2), 2.63\left(\mathrm{~s}, 2 \mathrm{H}, \mathrm{CH}_{2}\right.$ at $\left.\mathrm{C}-7\right), 7.13-7.55(\mathrm{~m}, 5 \mathrm{H}, \mathrm{Ph}-\mathrm{H}), 8.83$ $(\mathrm{s}, 1 \mathrm{H}, \mathrm{CH}$ at $\mathrm{C}-5) ;{ }^{13} \mathrm{C}$ NMR $\left(100 \mathrm{MHz}, \mathrm{DMSO}-d_{6}\right) \delta 14.41$, 24.42, 27.90, 36.42, 38.87, 50.15, 112.99, 119.22, 125.88, $126.67,128.30,129.20,132.43,140.64,144.52,159.05$, 194.70; EIMS $\mathrm{m} / \mathrm{z} 305.2\left(\mathrm{M}^{+}\right), 299,179.1$, calcd for $\mathrm{C}_{19} \mathrm{H}_{19} \mathrm{~N}_{3} \mathrm{O}$ 305.37; Anal. calcd for $\mathrm{C}_{19} \mathrm{H}_{19} \mathrm{~N}_{3} \mathrm{O}$ : C, 74.73; $\mathrm{H}$, 6.27; N, 13.76; found: C, 74.66; H, 6.35, N, 13.82\%.

\section{2-Phenyl-8,9-dihydropyrazolo[1,5-a]quinazolin-6(7H)-one} (6e): Pale yellow crystals, $215 \mathrm{mg}$ (82\% yield); mp 197-198 ${ }^{\circ} \mathrm{C} ;{ }^{1} \mathrm{H}$ NMR $\left(400 \mathrm{MHz}, \mathrm{DMSO}-d_{6}\right) \delta 2.25\left(\mathrm{~m}, 2 \mathrm{H}, \mathrm{CH}_{2}\right.$ at $\mathrm{C}-8), 2.64\left(\mathrm{t}, J=5.6 \mathrm{~Hz}, 2 \mathrm{H}, \mathrm{CH}_{2}\right.$ at C-9), $3.41(\mathrm{t}, J=5.6 \mathrm{~Hz}$, $2 \mathrm{H}, \mathrm{CH}_{2}$ at C-7), 7.39 (br s, $1 \mathrm{H}, \mathrm{CH}$ at $\left.\mathrm{C}-3\right), 7.48(\mathrm{~m}, 3 \mathrm{H}$, $\mathrm{Ph}-\mathrm{H}), 8.08$ (d, J = 7.2 Hz, 2H, Ph-H), 8.78 (s, 1H, CH at C-5); ${ }^{13} \mathrm{C}$ NMR (100 MHz, DMSO- $\left.d_{6}\right) \delta 19.97,23.46,36.63,79.19$, 95.49, 114.10, 126.44, 129.0, 129.69, 131.85, 146.77, 149.69, 154.39, 157.60, 162.32, 194.84; EIMS $m / z 263.1\left(\mathrm{M}^{+}\right), 235.1$, 152.1, calcd. for $\mathrm{C}_{16} \mathrm{H}_{13} \mathrm{~N}_{3} \mathrm{O} 263.11$; Anal. calcd for $\mathrm{C}_{16} \mathrm{H}_{13} \mathrm{~N}_{3} \mathrm{O}$ : C, 72.99; H, 4.98; N, 15.96; found: C, 72.94; $\mathrm{H}$, $5.18 ; \mathrm{N}, 16.32 \%$. 
8,8-Dimethyl-2-phenyl-8,9-dihydropyrazolo $[1,5-a]$ quinazolin-6(7H)-one (6f): Pale yellow crystals, $242 \mathrm{mg}(83 \%$ yield); mp 244-245 ${ }^{\circ} \mathrm{C} ;{ }^{1} \mathrm{H}$ NMR (400 MHz, DMSO- $d_{6}$ ) $\delta 1.18$ (s, 6H, $\left.2 \mathrm{CH}_{3}\right), 2.59$ (s, 2H, $\mathrm{CH}_{2}$ at C-9), 3.44 (s, 2H, $\mathrm{CH}_{2}$ at $\mathrm{C}-7), 7.34$ (s, 1H, CH at C-3), 7.50 (m, 3H, Ph-H), 8.09 (m, 2H, $\mathrm{Ph}-\mathrm{H}), 8.81$ (s, $1 \mathrm{H}, \mathrm{CH}$ at $\mathrm{C}-5) ;{ }^{13} \mathrm{C}$ NMR (100 MHz, DMSO$\left.d_{6}\right) \delta 28.47,32.73,37.17,50.86,95.94,113.79,127.02,129.29$, 129.97, 132.53, 146.90, 150.61, 152.87, 158.37, 194.85; Anal. calcd for $\mathrm{C}_{18} \mathrm{H}_{17} \mathrm{~N}_{3} \mathrm{O}$ : C, 74.20; H, 5.88; N, 14.42; found: $\mathrm{C}$, 74.32; H, 5.91; N, 14.44\%.

2-Hydroxy-8,8-dimethyl-3-(phenyldiazenyl)-8,9-dihydropyrazolo[1,5-a]quina-zolin-6(7H)-one (6g): Orange crystals, 295 mg (88\% yield); mp 254-255 ${ }^{\circ} \mathrm{C} ;{ }^{1} \mathrm{H}$ NMR (400 MHz, DMSO$\left.d_{6}\right) \delta 1.14\left(\mathrm{~s}, 6 \mathrm{H}, 2 \mathrm{CH}_{3}\right), 2.66\left(\mathrm{~s}, 2 \mathrm{H}, \mathrm{CH}_{2}\right.$ at $\left.\mathrm{C}-9\right), 3.26(\mathrm{~s}, 2 \mathrm{H}$, $\mathrm{CH}_{2}$ at C-7), $7.45(\mathrm{t}, J=7.2 \mathrm{~Hz}, 1 \mathrm{H}, \mathrm{Ph}-\mathrm{H}), 7.55$ (t, $J=7.6 \mathrm{~Hz}$, 2H, Ph-H), 7.85 (d, J=7.6 Hz, 2H, Ph-H), 8.95 (s, 1H, CH at $\mathrm{C}-5) ;{ }^{13} \mathrm{C}$ NMR (100 MHz, DMSO-d $\left.d_{6}\right) \delta 27.96,32.25,36.44$, 50.14, 79.20, 115.14, 115.74, 121.33, 129.34, 129.80, 144.26, 148.99, 151.95, 152.61, 162.10, 194.3; EIMS $m / z$ 335.1 $\left(\mathrm{M}^{+}\right)$, 307.1, 258.1, calcd for $\mathrm{C}_{18} \mathrm{H}_{17} \mathrm{~N}_{5} \mathrm{O}_{2}$ 335.14; Anal. calcd for $\mathrm{C}_{18} \mathrm{H}_{17} \mathrm{~N}_{5} \mathrm{O}_{2}$ : C, 64.47; 5.11; 20.88; found: $\mathrm{C}, 64.43 ; 5.33$; $20.95 \%$.

\section{Synthesis of (Z)-5,5-dimethyl-3-[(3-methyl- $1 H$-pyrazol-5-yl)amino]cyclohexanone (5)}

A solution of 1a $(1 \mathrm{mmol})$ and $\mathbf{2 a}(1 \mathrm{mmol})$ in DMF $(10 \mathrm{~mL})$ was heated under reflux in a Milestone Microwave Labstation at $150{ }^{\circ} \mathrm{C}$ for $10 \mathrm{~min}$. After concentration and cooling to room temperature, the resulting solid product so formed was collected by filtration, washed well with EtOH, dried and recrystallized from EtOH to afford a pure sample of compound 5 as yellow crystals, $186 \mathrm{mg}$ (85\% yield); mp $233-235^{\circ} \mathrm{C}$.

Synthesis of 4a: A solution of $1 \mathrm{a}(1 \mathrm{mmol})$ and $2 \mathrm{a}(1 \mathrm{mmol})$ in DMF $(10 \mathrm{~mL})$ was heated under reflux in a Milestone Microwave Labstation at $150{ }^{\circ} \mathrm{C}$ for $10 \mathrm{~min}$. After concentration and cooling to room temperature, the resulting solid product so formed was collected by filtration, washed well with EtOH, dried and recrystallized from EtOH to afford a pure sample of (Z)-3,3-dimethyl-5-(3-methyl-1H-pyrazol-5ylimino)cyclo-hexanone (5) as yellow crystals, $186 \mathrm{mg}(85 \%$ yield); mp 233-235 ${ }^{\circ} \mathrm{C}$.

Reaction of 5 with dimethylformamide dimethylacetal (DMFDMA, 3): A solution of 5 (1 mmol) and DMFDMA (3) $(1 \mathrm{mmol})$ in DMF $(10 \mathrm{~mL})$ was heated under reflux in a Milestone Microwave Labstation at $150{ }^{\circ} \mathrm{C}$ for $10 \mathrm{~min}$. After evaporation to dryness under reduced pressure, the resulting solid product was collected by filtration, washed well with EtOH, dried and recrystallized from $\mathrm{EtOH}$ to give $\mathbf{4 a}$.
Alternative synthesis of $6 \mathrm{~g}$ : Synthesis of 2-((3-hydroxy-4(phenyldiazenyl)-1H-pyrazol-5-ylamino)methylene)-5,5dimethylcyclohexane-1,3-dione (8): A solution of 1 f (1 $\mathrm{mmol})$, enaminone $7(1 \mathrm{mmol})$ in DMF $(10 \mathrm{~mL})$ was heated under reflux in a Milestone Microwave Labstation at $150{ }^{\circ} \mathrm{C}$ for $2 \mathrm{~min}$. After concentration and cooling to room temperature, the precipitated product was collected by filtration, washed well with $\mathrm{EtOH}$, dried and recrystallized from $\mathrm{EtOH}$ to give a pure sample of 8 as orange crystals, $303 \mathrm{mg}$ (88\% yield); mp 255-256 ${ }^{\circ} \mathrm{C}$; ${ }^{1} \mathrm{H}$ NMR (400 MHz, DMSO- $\left.d_{6}\right) \delta 1.01(\mathrm{~s}, 6 \mathrm{H}, 2$ $\left.\mathrm{CH}_{3}\right), 2.40\left(\mathrm{~s}, 2 \mathrm{H}, \mathrm{CH}_{2}\right), 3.26\left(\mathrm{~s}, 2 \mathrm{H}, \mathrm{CH}_{2}\right), 7.24-7.85(\mathrm{~m}, 6 \mathrm{H}$, $5 \mathrm{Ph}-\mathrm{H}$ and $\mathrm{CH}-\mathrm{NH}), 11.76(\mathrm{~s}, 1 \mathrm{H}, \mathrm{NH}), 12.59$ (s, 1H, pyrazole $\mathrm{NH}) ;{ }^{13} \mathrm{C}$ NMR (100 MHz, DMSO- $\left.d_{6}\right) \delta 27.95,30.70,50.12$, 109.66, 115.16, 115.74, 121.31, 126.16, 129.32, 129.64, 129.80, 144.34, 148.97, 152.57, 158.40, 194.23, 195.33; EIMS $\mathrm{m} / \mathrm{z}$ $353.2\left(\mathrm{M}^{+}\right)$, 335.1, 242.1, calcd. for $\mathrm{C}_{18} \mathrm{H}_{19} \mathrm{~N}_{5} \mathrm{O}_{3}$ 353.15; Anal. calcd for $\mathrm{C}_{18} \mathrm{H}_{19} \mathrm{~N}_{5} \mathrm{O}_{3}: \mathrm{C}, 61.18 ; \mathrm{H}, 5.42 ; \mathrm{N}, 19.82$; found: $\mathrm{C}$, 61.23; H, 5.45; N, 19.92\%.

Cyclization of 8. A solution of 8 ( $1 \mathrm{mmol})$ in DMF $(10 \mathrm{ml})$ was heated under reflux in a Milestone Microwave Labstation at 150 ${ }^{\circ} \mathrm{C}$ for $13 \mathrm{~min}$. The reaction mixture was evaporated to dryness in vacuo. The precipitated solid product was filtered off, washed with a small amount of EtOH, dried and recrystallized from $\mathrm{EtOH}$ to give an analytical pure sample of $\mathbf{6} \mathbf{g}$ (identical with an authentic sample, MS, ${ }^{1} \mathrm{H}$ NMR and ${ }^{13} \mathrm{C}$ NMR).

\section{Acknowledgements}

K. U. Sadek is grateful to the Alexander von Humboldt Foundation for donation of a Milestone START Microwave Labstation, which was of great help in finishing this work. M. H. Elnagdi and Moustafa Sherief Moustafa are grateful to Kuwait University Research Administration for the financial support of project $\mathrm{SC} 1 / 10$, and the analytical facilities provided by SAF projects No. GS 03/08 (Single crystal X-ray crystallography-Rigaku Rapid II) \& GS 01/01 \& GS 01/03 \& GS 01/05 are greatly appreciated.

\section{References}

1. Kessler, M.; Baudry, M.; Lynch, G. Brain Res. 1989, 489, 377. doi:10.1016/0006-8993(89)90875-5

2. McQuaid, L. A.; Smith, E. C. R.; South, K. K.; Mitch, C. H.; Schoepp, D. D.; True, R. A.; Calligaro, D. O.; O’Malley, P. J.; Lodge, D.; Ornstein, P. L. J. Med. Chem. 1992, 35, 3319. doi:10.1021/jm00096a002

3. Orvieto, F.; Branca, D.; Giomini, C.; Jones, P.; Koch, U.; Ontoria, J. M.; Palumbi, M. C.; Rowley, M.; Toniatti, C.; Muraglia, E. Bioorg. Med. Chem. Lett. 2009, 19, 4196. doi:10.1016/j.bmcl.2009.05.113

4. Watson, C. Y.; Whish, W. J. D.; Threadgill, M. D. Bioorg. Med. Chem. 1998, 6, 721. doi:10.1016/S0968-0896(98)00029-7

5. Weber, L.; Illgen, K.; Almstetter, M. Synlett 1999, 366. doi:10.1055/s-1999-2612 
6. Kantevari, S.; Vuppalapati, S. V. N.; Nagarapu, L. Catal. Commun. 2007, 8, 1857. doi:10.1016/j.catcom.2007.02.022

7. Sami, S.; Nandi, G. C.; Kumar, R.; Singh, M. S. Tetrahedron Lett. 2009, 50, 7096. doi:10.1016/j.tetlet.2009.10.022

8. Zhu, J.; Bienaymé, H., Eds. Multicomponent Reactions; Wiley-VCH: Weinheim, Germany, 2005.

9. Dömling, A.; Ugi, I. Angew. Chem., Int. Ed. 2000, 39, 3168. doi:10.1002/1521-3773(20000915)39:18<3168::AID-ANIE3168>3.0.CO ;2-U

10. Simon, C.; Constantieux, T.; Rodriguez, J. Eur. J. Org. Chem. 2004, 4957. doi:10.1002/ejoc.200400511

11. Carey, F. A.; Sundberg, R. J. Advanced Organic Chemistry, Part A, 5th ed.; Springer: New York, 2007.

12. Erkkilä, A.; Majander, I.; Pihko, P. M. Chem. Rev. 2007, 107, 5416. doi:10.1021/cr068388p

13. Laschat, S.; Becheanu, A.; Bell, T.; Baro, A. Synlett 2005, 2547. doi:10.1055/s-2005-918922

14. Kappe, C. O. Angew. Chem., Int. Ed. 2004, 43, 6250. doi:10.1002/anie.200400655

15. de la Hoz, A.; Díaz-Ortiz, Á.; Moreno, A. Chem. Soc. Rev. 2005, 34 , 164. doi:10.1039/b411438h

16. Bonrath, W.; Paz Schmidt, R. A. Ultrasound in Synthetic Organic Chemistry. In Advances in Organic Synthesis; Atta-ur-Rahman, Ed.; Bentham Sciences: Oak Park, IL, 2005; Vol. 1, pp 81-117.

17. Gravotto, G.; Cintas, P. J. Chem. Soc. Rev. 2006, 35, 180. doi:10.1039/b503848k

18. Quiroga, J.; Mejía, D.; Insuasty, B.; Abonía, R.; Nogueras, M.; Sánchez, A.; Cobo, J.; Low, J. N. Tetrahedron 2001, 57, 6947. doi:10.1016/S0040-4020(01)00649-4

19. Chebanov, V. A.; Saraev, V. E.; Desenko, S. M.; Chernenko, V. N.; Shishkina, S. V.; Shishkin, O. V.; Kobzar, K. M.; Kappe, C. O. Org. Lett. 2007, 9, 1691. doi:10.1021/ol070411।

20. Chebanov, V. A.; Saraev, V. E.; Desenko, S. M.; Chernenko, V. N.; Knyazeva, I. V.; Groth, U.; Glasnov, T. N.; Kappe, C. O. J. Org. Chem. 2008, 73, 5110. doi:10.1021/j0800825c

21. Ghotekar, B. K.; Jachak, M. N.; Toche, R. B. J. Heterocycl. Chem. 2009, 46, 708. doi:10.1002/jhet.128

22. Sadek, K. U.; Shaker, R. M.; Abd Elrady, M.; Elnagdi, M. H. Tetrahedron Lett. 2010, 51, 6319. doi:10.1016/j.tetlet.2010.09.114

23. Abd El Latif, F. M.; Barsy, M. A.; Aref, A. M.; Sadek, K. U. Green Chem. 2002, 4, 196. doi:10.1039/b110723m

24. Mekheimer, R. A.; Sadek, K. U. J. Heterocycl. Chem. 2009, 46, 149. doi:10.1002/jhet.13

25. CCDC 825123 contains the supplementary crystallographic data for compound $\mathbf{6 a}$. These data can be obtained free of charge from the Cambridge Crystallographic Data Centre via http://www.ccdc.cam.ac.uk

26. CCDC 833076 contains the supplementary crystallographic data for compound 5. These data can be obtained free of charge from the Cambridge Crystallographic Data Centre via http://www.ccdc.cam.ac.uk

27. CCDC 827653 contains the supplementary crystallographic data for compound $6 e$. These data can be obtained free of charge from the Cambridge Crystallographic Data Centre via http://www.ccdc.cam.ac.uk

28. CCDC 826742 contains the supplementary crystallographic data for compound $\mathbf{6 g}$. These data can be obtained free of charge from the Cambridge Crystallographic Data Centre via http://www.ccdc.cam.ac.uk
29. Elnagdi, M. H. Tetrahedron 1974, 30, 2791. doi:10.1016/S0040-4020(01)97447-2

\section{License and Terms}

This is an Open Access article under the terms of the Creative Commons Attribution License (http://creativecommons.org/licenses/by/2.0), which permits unrestricted use, distribution, and reproduction in any medium, provided the original work is properly cited.

The license is subject to the Beilstein Journal of Organic Chemistry terms and conditions:

(http://www.beilstein-journals.org/bjoc)

The definitive version of this article is the electronic one which can be found at:

doi:10.3762/bjoc. 8.3 